\section{Is chondroitin sulfate plus glucosamine superior to placebo in the treatment of knee osteoarthritis?}

We read with interest the article by Hochberg $e a^{1}{ }^{1}$ related to the efficacy and safety of chondroitin sulfate plus glucosamine hydrochloride $(\mathrm{CS}+\mathrm{GH})$ versus celecoxib in patients with knee osteoarthritis and severe pain. The study suggested that CS $+\mathrm{GH}$ has comparable efficacy to celecoxib in reducing pain, stiffness, functional limitation and joint swelling/effusion after 6 months with a good safety profile. These results were 'promising'. We really appreciate the work that was done by the authors. However, there are worthwhile issues that need to be explored.

The study was designed as a non-inferiority trial without placebo arm. The authors explained that, 'The use of a placebo group was not considered appropriate for ethical and methodological reasons'. We agree with this. The authors added that the use of placebo arm was not considered necessary as the Glucosamine/Chondroitin Arthritis Intervention Trial (GAIT) already compared both active treatments $(\mathrm{CS}+\mathrm{GH}$ and celecoxib) with placebo and came to a conclusion that a subset of participants with more severe baseline pain appeared to benefit by use of $\mathrm{CS}+\mathrm{GH}$ as compared with placebo. ${ }^{2}$ However, a subsequent GAIT report clearly pointed out that none of the interactions between severe pain and treatment groups were statistically significant over a follow-up of 24 months. ${ }^{3}$ In addition, the authors did not mention the Long-term Evaluation of Glucosamine Sulfate (LEGS) study that was published in January $2014 .{ }^{4}$ The LEGS study reported that although the glucosamine-chondroitin combination demonstrated reduced knee pain over the study period, it did not show significant symptomatic benefit above placebo. Furthermore, the authors also indicated that the results of their study were in accordance with data from three other studies, ${ }^{2} 56$ including the original GAIT for the combination. However, it should be noted that the combination group in two of the three studies contained the CS $+\mathrm{GH}$ and the manganese ascorbate. ${ }^{5} 6$ The therapeutic efficacy of the combination could probably be improved by the manganese ascorbate. Thus, the efficacy of $\mathrm{CS}+\mathrm{GH}$ versus placebo in the treatment of knee osteoarthritis is still unclear. In such cases, we are not sure whether it is appropriate to conduct a noninferiority trial without placebo control to examine the efficacy of CS +GH and celecoxib for painful knee osteoarthritis.

The use of placebo control is a standard group in a large number of clinical trials. In consideration of the effects from active treatment that do not depend entirely upon itself, the objective of the placebo control is to account for the placebo effect. Because of the heavily negative connotations of the very words 'placebo effect', Benson et $a l^{7}$ even suggested that the term should be replaced by 'remembered wellness' which should not be belittled or ridiculed. Another systematic review of 130 trials also reported that the placebo has possible small benefits in studies with continuous subjective outcomes and for the treatment of pain. ${ }^{8}$

Above all, without a placebo group to compare against, it is a little hard to know whether the active treatments $(\mathrm{CS}+\mathrm{GH}$ and celecoxib) themselves had any effect. We respect the great contributions of the authors and we are very much looking forward to the follow-up results of this study.

\section{Chao Zeng, ${ }^{1}$ Jie Wei, ${ }^{2}$ Guang-hua Lei ${ }^{1}$}

${ }^{1}$ Department of Orthopaedics, Xiangya Hospital, Central South University, Changsha, Hunan Province, China

${ }^{2}$ Department of Epidemiology and Health Statistics, School of Public Health, Central South University, Changsha, Hunan Province, China.

Correspondence to Professor Guang-hua Lei, Department of Orthopaedics, Xiangya Hospital, Central South University, Xiangya Road, Changsha, Hunan Province 410008, China; Igh9640@sina.cn

Contributors CZ: concept, writing; JW: writing; and G-hL: concept, revising. Competing interests None.

Provenance and peer review Not commissioned; internally peer reviewed.

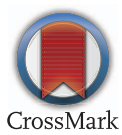

To cite Zeng Co, Wei J, Lei G-hua. Ann Rheum Dis 2015;74:e37.

Accepted 17 February 2015

Published Online First 6 March 2015

Ann Rheum Dis 2015;74:e37. doi:10.1136/annrheumdis-2015-207476

\section{REFERENCES}

1 Hochberg MC, Martel-Pelletier J, Monfort J, et al., on behalf of the MOVES Investigation Group. Combined chondroitin sulfate and glucosamine for painful knee osteoarthritis: a multicenter, randomised, double-blind, non-inferiority trial versus placebo. Ann Rheum Dis 2015. Published Online First: 14 Jan 2015. doi:10.1136/ annrheumdis-2014-206792

2 Clegg DO, Reda DJ, Harris CL, et al. Glucosamine, chondroitin sulfate, and the two in combination for painful knee osteoarthritis. N Eng/ J Med 2006;354:795-808.

3 Sawitzke AD, Shi $H$, Finco MF, et al. Clinical efficacy and safety of glucosamine, chondroitin sulphate, their combination, celecoxib or placebo taken to treat osteoarthritis of the knee: 2-year results from GAIT. Ann Rheum Dis 2010:69:1459-64.

4 Fransen $\mathrm{M}$, Agaliotis $\mathrm{M}$, Nairn L, et al. Glucosamine and chondroitin for knee osteoarthritis: a double-blind randomised placebo- controlled clinical trial evaluating single and combination regimens. Ann Rheum Dis 2015;74:851-8.

5 Leffler CT, Philippi AF, Leffler SG, et al. Glucosamine, chondroitin, and manganese ascorbate for degenerative joint disease of the knee or low back: a randomized, double-blind, placebo-controlled pilot study. Mil Med 1999;164:85-91.

6 Das A Jr, Hammad TA. Efficacy of a combination of FCHG49 glucosamine hydrochloride, TRH122 low molecular weight sodium chondroitin sulfate and manganese ascorbate in the management of knee osteoarthritis. Osteoarthritis Cartilage 2000;8:343-50.

7 Benson H, Friedman R. Harnessing the power of the placebo effect and renaming it 'remembered wellness'. Annu Rev Med 1996;47:193-9.

8 Hróbjartsson A, Gøtzsche PC. Is the placebo powerless? An analysis of clinical trials comparing placebo with no treatment. N Engl J Med 2001;344:1594-602. 